\title{
Effect of Lipid Emulsion on Stability of Ampicillin in Total Parenteral Nutrition
}

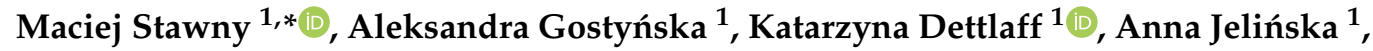 \\ Eliza Główka ${ }^{2}$ and Magdalena Ogrodowczyk ${ }^{1}$ \\ 1 Department of Pharmaceutical Chemistry, Poznan University of Medical Sciences, 6 Grunwaldzka, \\ 60-780 Poznań, Poland; aleksandra.gostynska@spsk2.pl (A.G.); dettlaff@ump.edu.pl (K.D.); \\ ajelinsk@ump.edu.pl (A.J.); mogrodo@ump.edu.pl (M.O.) \\ 2 Department of Pharmaceutical Technology, Poznan University of Medical Sciences, 6 Grunwaldzka, \\ 60-780 Poznań, Poland; eglowka@ump.edu.pl \\ * Correspondence: mstawny@ump.edu.pl; Tel.: +48-509-207-430
}

Received: 8 February 2019; Accepted: 28 February 2019; Published: 6 March 2019

\begin{abstract}
Background: Ampicillin (AMP) is frequently administered parenterally in critically ill patients with meningitis or endocarditis. Many of them require parallel infusion of total parenteral nutrition (TPN) admixtures. The aim of the study was to determine the physicochemical stability of AMP in TPN admixtures. Methods: AMP was added to two formulations of TPN admixtures differing in the lipid emulsion (Lipofundin ${ }^{\circledR}$ MCT /LCT $20 \%$ or LIPIDem ${ }^{\circledR}$ ). Samples were stored at $4 \pm 1{ }^{\circ} \mathrm{C}$ with light protection, and at $25 \pm 1{ }^{\circ} \mathrm{C}$ with and without light protection to assess the impact of temperature and light on formulation stability. Every $24 \mathrm{~h}$ the $\mathrm{pH}$, zeta potential, mean droplet diameter (MDD) of a lipid emulsion, and AMP concentration using HPLC method were determined. The assessment of stability and compatibility of TPN admixtures with vitamins and trace elements was carried out immediately after preparation and after $24 \mathrm{~h}$ of storage. Results: The addition of AMP as well as vitamins and trace elements to the TPN admixtures did not affect their physical stability. An increase in the $\mathrm{pH}$ value of approx. 0.6 and reduction of zeta potential were observed. The MDD of the lipid emulsions was below the limit of $500 \mathrm{~nm}$ (dynamic light scattering (DLS) method) and no fat droplets greater than $525 \mathrm{~nm}$ were observed (light diffraction (LD) method). The content of AMP after the first $24 \mathrm{~h}$ was within the acceptable limit of $90 \%$ for TPN admixtures stored at $4 \pm 1{ }^{\circ} \mathrm{C}$ and $25 \pm 1{ }^{\circ} \mathrm{C}$ with light protection. Conclusions: The results showed that co-administration of AMP in the same bag with TPN admixture at the tested dose is possible when used ex tempore and with light protection.
\end{abstract}

Keywords: total parenteral nutrition; ampicillin; physicochemical stability; lipid emulsion

\section{Introduction}

Ampicillin (AMP) is a $\beta$-lactam antibiotic with a wide range of activity often used in clinical practice. High doses of AMP are frequently administered parenterally in critically ill patients with meningitis or endocarditis. Such patients often receive concomitantly an intravenous infusion of total parenteral nutrition (TPN) admixtures in order to maintain a proper nutritional status and to alleviate the metabolic response resulting from stress, injury, or extensive surgery. In some cases, co-administration of drugs in the same infusion with TPN is needed due to a limitation of available intravenous (IV) lines. There are few reports in the literature regarding physicochemical compatibility and stability of TPN admixtures and IV drugs. However, in recent years this topic has attracted more attention [1-5]. Administration of a drug with unknown stability is a direct threat to a patient's health and life. It is particularly important to confirm the possibility of using medications in parallel 
with other medicines via Y-site or adding them to a medium not described in the SPC (Summaries of Product Characteristics) [6]. TPN admixture is a compounded drug resulting from the combination of amino acid, glucose, electrolyte, and lipid emulsion. The determination of an IV medication's stability in this kind of medium is very problematic and requires appropriate physicochemical assessment proving stability and compatibility of both TPN admixtures and the added drug [7-9].

In this study, physical stability studies of TPN admixtures were conducted to determine the effect of AMP, time, and storage conditions on formulation stability. For this purpose, we assessed such physical parameters as $\mathrm{pH}$, mean droplet diameter (MDD) of the lipid emulsions, and zeta potential of TPN admixtures with and without AMP. Regarding the chemical stability of AMP, high-performance liquid chromatography (HPLC) was performed.

To the best of our knowledge, so far there have been no studies confirming the possibility of adding AMP to TPN admixtures in adults. Therefore, we decided to investigate this scenario. The aim of the study was to determine the physicochemical compatibility and stability of AMP in two standard TPN admixtures containing different types of lipid emulsion, which is key to ensure patients' safety.

\section{Materials and Methods}

\subsection{Compositions of TPN Admixtures}

In this study, we chose two compositions of TPN admixtures differing in the type of lipid source (Table 1). TPN admixtures composition was based on the literature recommendations for adult hospitalized patients and calculated for a patient weighing $60 \mathrm{~kg}$ [10]. The only difference between studied compositions was the type of lipid emulsion. Lipofundin ${ }^{\circledR} \mathrm{MCT} / \mathrm{LCT}$-containing TPN admixtures (A, A + AMP) consisted of a lipid emulsion based on long-chain (LCT) and medium-chain $(\mathrm{MCT})$ triglycerides. In contrast, the LIPIDem ${ }^{\circledR}$-containing TPN admixtures $(\mathrm{B}, \mathrm{B}+\mathrm{AMP})$ were based on a lipid emulsion consisting of MCT, LCT, and triglycerides of omega-3 acids. For a 7-day stability study, we prepared TPN admixtures without vitamins and trace elements, as in clinical practice these components, due to their low stability, are added just before administration to patients. Therefore, the physical stability studies of TPN admixtures (A, A + AMP, B, and B + AMP) with vitamins (one vial of Cernevit, Baxter SA, Lessines, Belgium) and trace elements (one ampoule of Tracutil, B. Braun Melsungen AG, Germany) were carried out only on the day of preparation of the TPN admixtures and after $24 \mathrm{~h}$.

Table 1. Composition of TPN admixtures.

\begin{tabular}{|c|c|c|c|c|}
\hline \multirow{2}{*}{ Ingredients } & \multirow{2}{*}{ Pharmaceutical Preparation } & \multirow{2}{*}{ Unit } & \multicolumn{2}{|c|}{ Composition of TPN Admixtures } \\
\hline & & & $\mathbf{A}$ & B \\
\hline Amino acid & $\begin{array}{c}\text { Aminoplasmal }^{\circledR} \text { B. Braun } 10 \% \text { E } \\
\text { (B. Braun Melsungen AG, Germany) }\end{array}$ & \multirow{5}{*}{$\mathrm{mL}$} & 600 & 600 \\
\hline Carbohydrates & $\begin{array}{l}\quad 40 \% \text { Glucose B. Braun } \\
\text { (B. Braun Melsungen AG, Germany) }\end{array}$ & & 550 & 550 \\
\hline \multirow{2}{*}{ Lipids } & $\begin{array}{c}\text { Lipofundin }^{\circledR} \text { MCT /LCT 20\% } \\
\text { (B. Braun Melsungen AG, Germany) }\end{array}$ & & 300 & 0 \\
\hline & $\begin{array}{c}\text { LIPIDem }^{\circledR} \\
\text { (B. Braun Melsungen AG, Germany) }\end{array}$ & & 0 & 300 \\
\hline Water & $\begin{array}{c}\text { Aqua ad iniectabile } \\
\text { (B. Braun Melsungen AG, Germany) }\end{array}$ & & 902 & 902 \\
\hline Sodium & $\begin{array}{c}\text { Natrium Chloratum 10\% } \\
\text { (B. Braun Melsungen AG, Germany) }\end{array}$ & & 102 & 102 \\
\hline
\end{tabular}


Table 1. Cont.

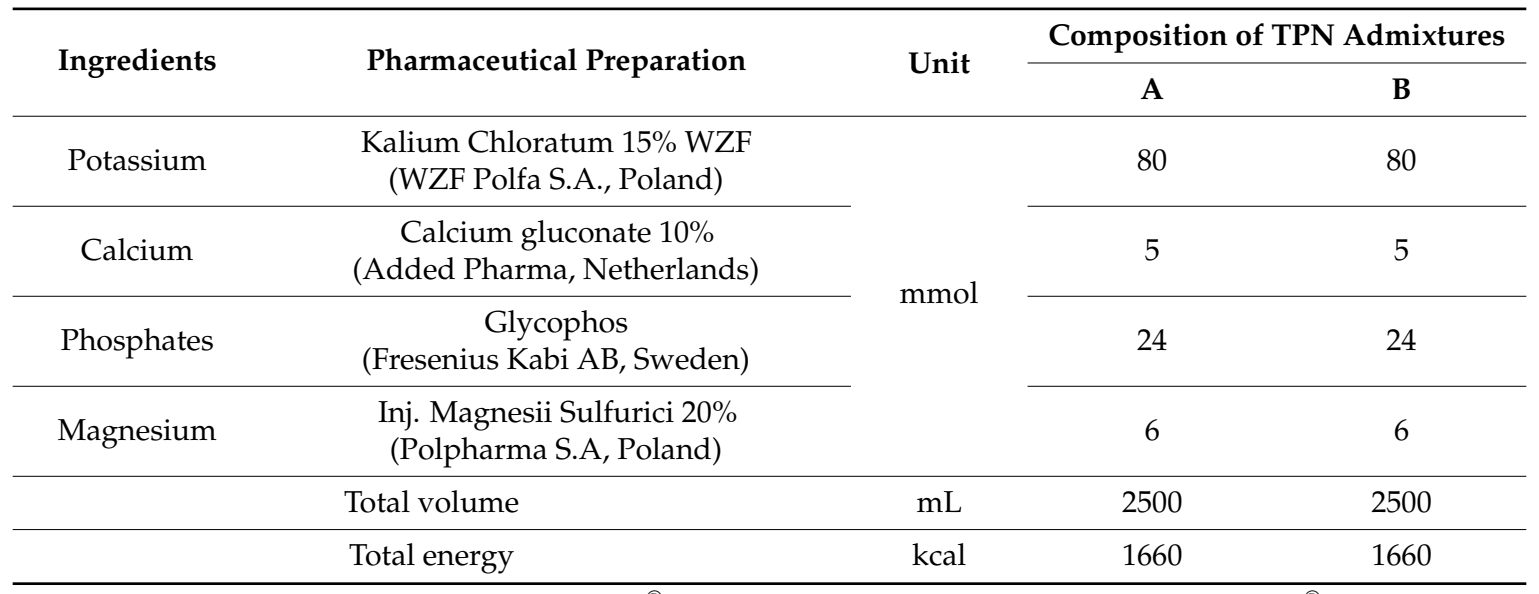

TPN: total parenteral nutrition; A: Lipofundin ${ }^{\circledR}$ MCT/LCT-containing TPN admixture; B: LIPIDem ${ }^{\circledR}$-containing TPN admixture.

\subsection{TPN Admixtures and Samples Preparation}

The TPN admixtures were prepared in aseptic conditions under a laminar-flow air hood using a Pinnacle B. Braun automatic compounder (B. Braun Melsungen AG, Germany). All doses were calculated as one-tenth of standard daily dose. The final volume of $250 \mathrm{~mL}$ was packaged in an ethylene-vinyl acetate (EVA) bag with a capacity of $300 \mathrm{~mL}$ (B. Braun Melsungen AG, Germany).

For sample preparation, a dose of $1 \mathrm{~g}$ of AMP was chosen as one-tenth of the standard dose used for serious bacterial infections like bacterial meningitis or endocarditis. The maximum dose of AMP for adult patients is $14 \mathrm{~g}$ per day. The tested materials were TPN admixtures mixed with $1 \mathrm{~g}$ of AMP (Ampicillin TZF $1000 \mathrm{mg}$ ) previously dissolved in $10 \mathrm{~mL}$ of water for injection (A + AMP, B + AMP) and reference samples were TPN admixtures without the addition of the drug (A, B). Each admixture was prepared separately in triplicate.

The A + AMP admixtures were stored at $4 \pm 1{ }^{\circ} \mathrm{C}$ with light protection, and at $25 \pm 1{ }^{\circ} \mathrm{C}$ with and without light protection. The other TPN admixtures (A, B, and B + AMP) were stored at $4 \pm 1{ }^{\circ} \mathrm{C}$ with light protection. Physicochemical analysis was performed on the day of admixture preparation and after $24 \mathrm{~h}, 48 \mathrm{~h}, 72 \mathrm{~h}, 92 \mathrm{~h}, 120 \mathrm{~h}$, and $144 \mathrm{~h}$. Samples were aseptically collected from each TPN admixture at appropriate intervals using a plastic syringe for the physicochemical tests. Every $24 \mathrm{~h}$, a volume of $10.0 \mathrm{~mL}$ was withdrawn from each TPN admixture and divided into two samples. For chemical stability tests, $3.0 \mathrm{~mL}$ was used, and the rest was used for physical stability assays (visual inspection, $\mathrm{pH}$, mean droplet diameter, and zeta potential determination).

Additionally, for the tested TPN admixtures (A, B, A + AMP, B + AMP) with vitamins and trace elements, the distribution of large-diameter droplet sizes assessment was performed in accordance with the requirements of the US Pharmacopoeia [11]. The measurements were taken immediately after the preparation of the samples and after $24 \mathrm{~h}$. A routine gravity infusion set (Exadrop, B. Braun AG Melsungen) was used for sample collection, a procedure simulating the administration of TPN admixtures to hospital patients. All samples were stored at $25 \pm 1{ }^{\circ} \mathrm{C}$ with light protection. A diagram of the study design is presented in Figure 1. 


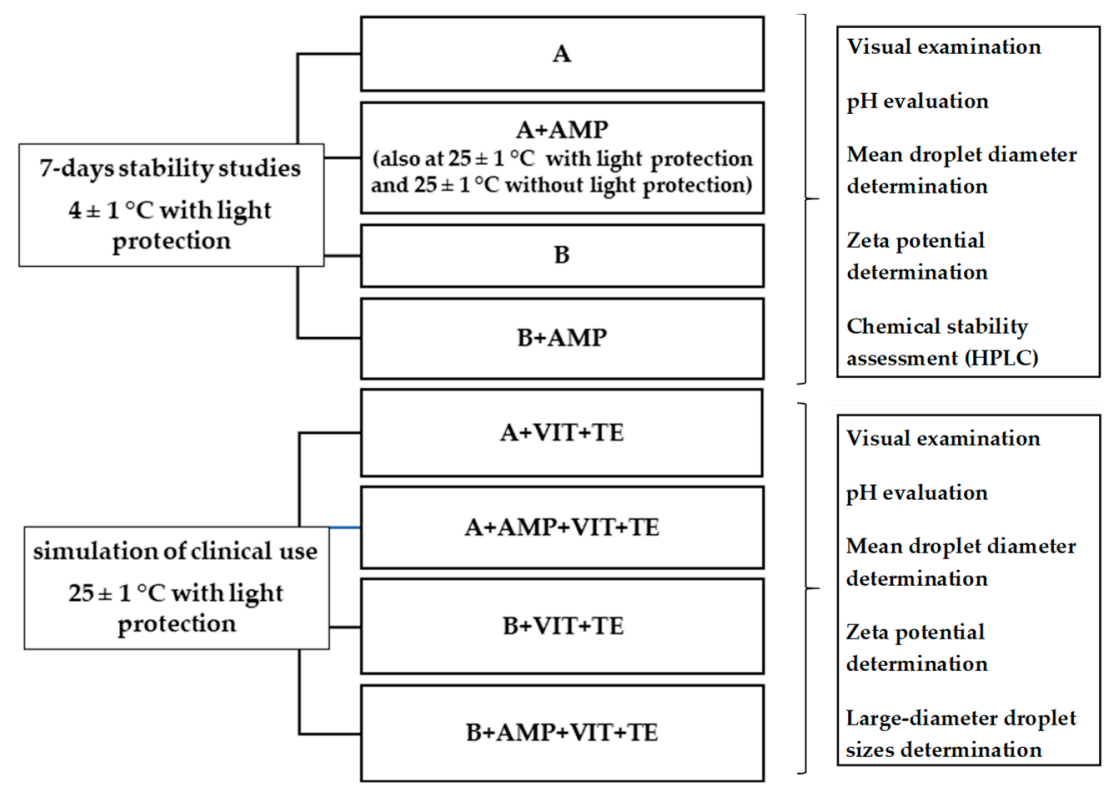

Figure 1. Diagram of study design. TPN: total parenteral nutrition; A: Lipofundin ${ }^{\circledR}$ MCT/LCT-containing TPN admixture without ampicillin; B: LIPIDem ${ }^{\circledR}$-containing TPN admixture without ampicillin; A + AMP, B + AMP: TPN admixtures with ampicillin; A + VIT + TE, B + VIT + TE: TPN admixtures with vitamins and trace elements; A + AMP + VIT + TE, B + AMP + VIT + TE: TPN admixtures with ampicillin, vitamins, and trace elements.

\subsection{Physical Stability}

\subsubsection{Visual Examination}

In accordance with the European Pharmacopoeia [12], all samples were visually assessed for the presence of particles and color change. Visual inspection was performed against a black-and-white contrast background by two observers.

\subsection{2. $\mathrm{pH}$ Evaluation}

For the evaluation of $\mathrm{pH}$, a Mettler Toledo Seven Compact $\mathrm{pH} /$ ion $\mathrm{S} 220^{\circledR} \mathrm{pH}$-meter was used. The $\mathrm{pH}$ was measured by dipping the electrode directly into the TPN admixture, at room temperature. The $\mathrm{pH}$ of each sample was measured in triplicate.

\subsubsection{Mean Droplet Diameter and Zeta Potential Determination}

The intensity weighted mean droplet diameter (MDD) of lipid emulsion, polydispersity index (PDI), and zeta potential of TPN admixtures were measured at $25^{\circ} \mathrm{C}$ using a Zetasizer Nano ZS (Malvern Instruments, Malvern, UK) by dynamic light scattering (DLS) and laser Doppler velocimetry (LDV), respectively. A dip cell (zen1002, Malvern Instruments) with a pair of parallel Pd electrodes was used to provide electrical trigger on charged particles. The signals were collected at an angle of $12.8^{\circ}$ and the data were analyzed using Zetasizer Software. As suggested by ISO13099 [13,14], the Smoluchowski model was used to calculate zeta potential values of nanoparticles in aqueous media. Samples for size and zeta potential measurement were prepared in accordance to the same protocol. One milliliter of tested TPN admixtures or reference samples was diluted 10 times with water for injection and then $1 \mathrm{~mL}$ of diluted sample was transferred to a Malvern Clear Zeta Potential cell for DLS and LDV measurements. 


\subsubsection{Large-Diameter Droplet Sizes Determination}

The percent of particles below $500 \mathrm{~nm}, 525 \mathrm{~nm}$, and above $5 \mu \mathrm{m}$ were estimated by light diffraction (LD) method based on Mie theory using Mastersizer ${ }^{\circledR} 3000$ analyzer (Malvern Panalytical, Malvern, UK). The Hydro SV small volume wet sample dispersion unit was used and set at $1500 \mathrm{rpm}$. The analyzer typically enables particle size distribution from $10 \mathrm{~nm}$ to $3500 \mu \mathrm{m}$ to be measured depending on sample and sample preparation. The cuvette was filled with degassed distilled water passed through a filter with $0.2 \mu \mathrm{m}$ porosity and the background was measured. Undiluted samples $(15 \mu \mathrm{L})$ were added to the cuvette until obscuration was achieved in the range of $1 \%$ to $5 \%$. The refractive and absorption indices were set at 1.449 and 0.01 , respectively. Each sample was measured in triplicate.

\subsection{Chemical Stability Assessment}

An HPLC method was used for the quantitative determination of AMP in the TPN admixtures. The Merck Hitachi L-7100 HPLC system was used. The analytical column was a $250 \mathrm{~mm} \times 4.6 \mathrm{~mm}$ in diameter reverse phase C18 (LiChrospher ${ }^{\circledR} 100$, endcapped, $5 \mu \mathrm{m}$ ) coupled with 4-mm length pre-column reverse phase $\mathrm{C} 18$ (LiChrospher $\left.{ }^{\circledR} 100,5 \mu \mathrm{m}\right)$. As mobile phase solvent A (12\% acetic acid $0.5 \mathrm{~mL}, 0.2 \mathrm{M}$ potassium dihydrogenphosphate $50 \mathrm{~mL}$, acetonitrile $50 \mathrm{~mL}$, water ad $1000 \mathrm{~mL}$ ) and solvent $\mathrm{B}(12 \%$ acetic acid $0.5 \mathrm{~mL}, 0.2 \mathrm{M}$ potassium dihydrogenphosphate $50 \mathrm{~mL}$, acetonitrile $400 \mathrm{~mL}$, water ad $1000 \mathrm{~mL})$ at the ratio $95: 5(\mathrm{v} / \mathrm{v})$ were used. Separation was performed at $25^{\circ} \mathrm{C}$, the mobile phase flow was $1 \mathrm{~mL} / \mathrm{min}$, and the analytical wavelength was $230 \mathrm{~nm}$. Samples for HPLC analysis were prepared by mixing $3.0 \mathrm{~mL}$ of TPN admixture with $1.0 \mathrm{~mL}$ of chloroform in $10-\mathrm{mL}$ plastic vials. The vials were shaken for $10 \mathrm{~min}$ and then centrifuged for $15 \mathrm{~min}$ at a rate of $5800 \mathrm{rpm}$. Samples were collected from the centrifuged supernatant, filtered through a membrane filter (pore size $0.2 \mu \mathrm{m}$ ), and injected into a chromatographic column for HPLC analysis (sample volume $10 \mu \mathrm{L}$ ).

\subsection{Statistical Analysis}

The data were analyzed using Statistica 12 software (StatSoft Polska, Cracow, Poland). Repeated-measures analyses of variance (ANOVAs) were used to determine whether the drug content, storage conditions, or storage time had an effect on the $\mathrm{pH}$, the MDD of lipid emulsion, and the zeta potential. The a priori level of significance was $p<0.05$. In the case of a major effect or interaction, significant differences between the tested TPN admixtures (with drugs) and the reference samples under the study conditions were identified using Tukey's honest significant difference test post hoc tests. The significance was defined as $p<0.05$.

\section{Results}

\subsection{Physical Stability}

During the entire study, none of the tested samples showed the presence of particles or color change. Immediately after preparation $(\mathrm{t}=0 \mathrm{~h})$, the measured $\mathrm{pH}$ values of reference samples $(\mathrm{A}, \mathrm{B})$ were $6.33 \pm 0.02$ and $6.42 \pm 0.02$ for Lipofundin ${ }^{\circledR} \mathrm{MCT} /$ LCT-containing and LIPIDem ${ }^{\circledR}$-containing TPN admixtures, respectively (Figure 2). The addition of AMP increased significantly $(p<0.05)$ the $\mathrm{pH}$ by approx. 0.6 in both formulations (A + AMP, B + AMP). The $\mathrm{pH}$ values of Lipofundin ${ }^{\circledR}$ MCT/LCT-containing TPN admixtures with AMP decreased slightly but significantly in time regardless of the storage conditions (Figure 3). The same pattern was observed in the case of LIPIDem $^{\circledR}$-containing TPN admixtures with AMP (Figure 2). 

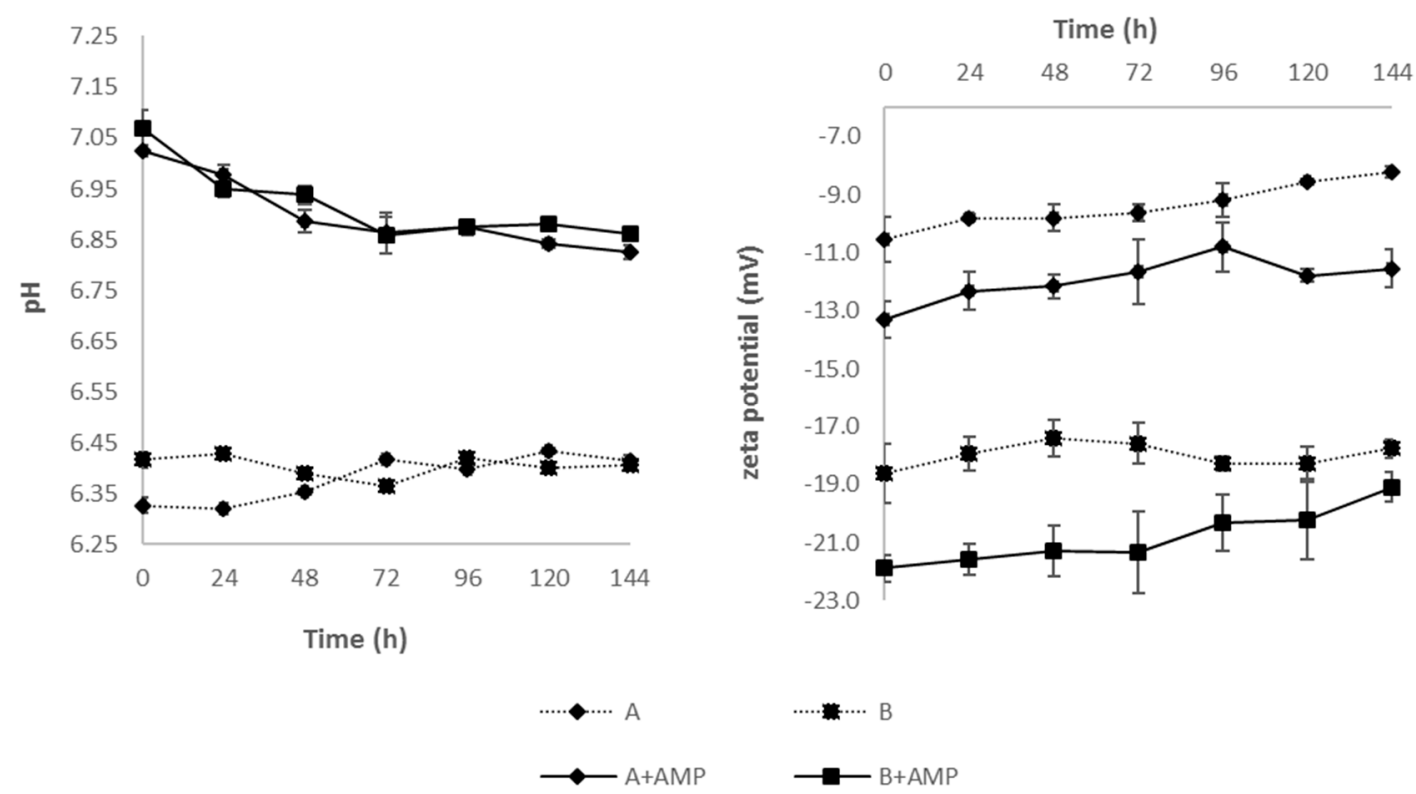

Figure 2. Results of $\mathrm{pH}$ and zeta potential of TPN admixtures without (A, B) and with (A + AMP, $\mathrm{B}+\mathrm{AMP}$ ) ampicillin during storage at $4 \pm 1^{\circ} \mathrm{C}$ with light protection. Results are shown as the mean values of three samples \pm SD.
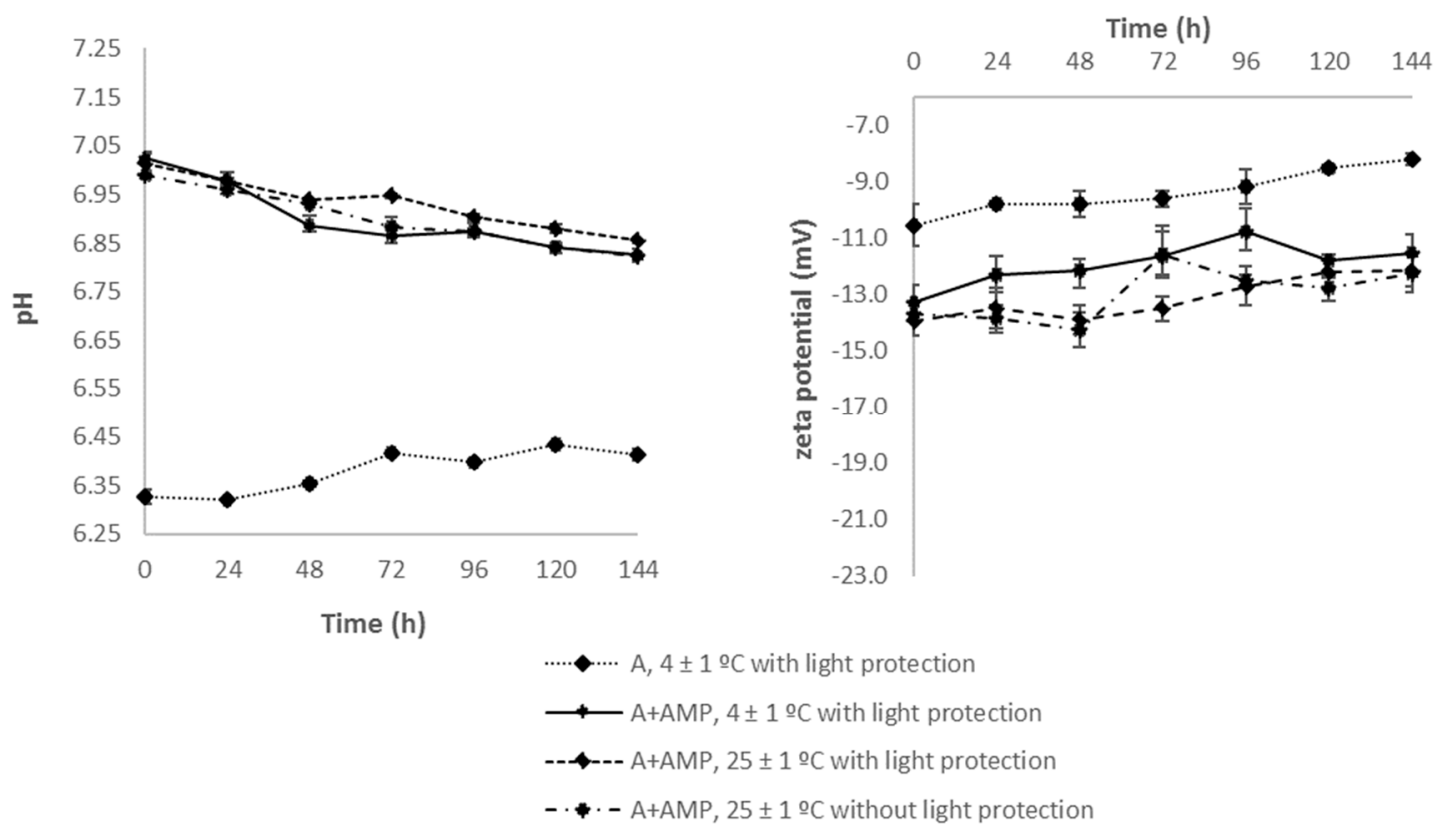

Figure 3. Values of $\mathrm{pH}$ and zeta potential of studied basic composition of TPN admixtures with (A + AMP) and without AMP (A) during storage at different conditions. Results are shown as the mean values of three samples $\pm \mathrm{SD}$.

Analyzing the results of the MDD of lipid emulsions in TPN admixtures, there was no statistically significant differences $(p>0.05)$ observed between TPN admixtures without AMP (A, B) and corresponding samples containing AMP (A + AMP, B + AMP) stored at $4 \pm 1{ }^{\circ} \mathrm{C}$ with light protection (Table 2). MDDs of the lipid emulsions in TPN admixtures without AMP (A, B) varied from $220.2 \pm 5.4 \mathrm{~nm}$ to $228.2 \pm 0.9 \mathrm{~nm}$ for Lipofundin ${ }^{\circledR}$ MCT/LCT-containing TPN admixtures, and $219.1 \pm 5.6 \mathrm{~nm}$ to $227.2 \pm 1.0 \mathrm{~nm}$ when LIPIDem ${ }^{\circledR}$ was used. The results showed that the lipid source was not associated with MDD values. The addition of AMP to the TPN admixtures did not cause any significant and characteristic changes in the MDD of the lipid emulsion particles, which remained 
below the limit of $500 \mathrm{~nm}$. The storage conditions also did not affect the MDD of the lipid emulsions of Lipofundin ${ }^{\circledR}$ MCT/LCT-containing TPN admixtures (Table 2).

Table 2. MDD of lipid emulsion in studied TPN admixtures during storage. Results are shown as the mean values of three samples $\pm \mathrm{SD}$.

\begin{tabular}{ccccccc}
\hline & \multicolumn{7}{c}{ MDD $\pm \mathbf{S D}$ (nm) } \\
\cline { 2 - 7 } Time (h) & $\mathbf{A}$ & $\mathbf{A}+\mathbf{A M P}$ & $\mathbf{A}+\mathbf{A M P}$ & $\mathbf{A}+\mathbf{A M P}$ & $\mathbf{B}$ & $\mathbf{B}+\mathbf{A M P}$ \\
\cline { 2 - 7 } & $\mathbf{4} \pm \mathbf{1}{ }^{\circ} \mathbf{C}$ & $\mathbf{4} \pm \mathbf{1}{ }^{\circ} \mathbf{C}$ & $\mathbf{2 5} \pm \mathbf{1}{ }^{\circ} \mathbf{C}$ & $\mathbf{2 5} \pm \mathbf{1}{ }^{\circ} \mathbf{C}$ & $\mathbf{4} \pm \mathbf{1}{ }^{\circ} \mathbf{C}$ \\
\cline { 2 - 7 } & With Light & With Light & With Light & Without Light & With Light & With Light \\
& Protection & Protection & Protection & Protection & Protection & Protection \\
\hline $\mathbf{0}$ & $220.2 \pm 5.4$ & $216.9 \pm 1.7$ & $220.3 \pm 1.2$ & $217.0 \pm 1.6$ & $219.5 \pm 4.5$ & $221.5 \pm 3.2$ \\
$\mathbf{2 4}$ & $219.3 \pm 2.2$ & $220.6 \pm 2.1$ & $215.3 \pm 3.9$ & $219.0 \pm 3.8$ & $219.1 \pm 5.6$ & $220.7 \pm 4.4$ \\
$\mathbf{4 8}$ & $218.5 \pm 0.8$ & $221.0 \pm 1.8$ & $219.0 \pm 1.2$ & $220.8 \pm 1.6$ & $219.6 \pm 4.6$ & $220.7 \pm 2.0$ \\
$\mathbf{7 2}$ & $219.4 \pm 1.8$ & $223.7 \pm 0.9$ & $220.8 \pm 4.3$ & $226.3 \pm 3.1$ & $221.2 \pm 5.3$ & $221.2 \pm 4.6$ \\
$\mathbf{9 6}$ & $225.5 \pm 5.9$ & $226.3 \pm 3.2$ & $224.3 \pm 8.3$ & $223.7 \pm 5.9$ & $222.8 \pm 0.3$ & $224.6 \pm 5.7$ \\
$\mathbf{1 2 0}$ & $226.6 \pm 3.3$ & $226.5 \pm 3.4$ & $227.8 \pm 4.6$ & $227.2 \pm 2.0$ & $227.2 \pm 1.0$ & $229.2 \pm 4.9$ \\
$\mathbf{1 4 4}$ & $228.2 \pm 0.9$ & $228.5 \pm 1.7$ & $226.1 \pm 3.7$ & $233.6 \pm 4.5$ & $225.5 \pm 0.1$ & $228.4 \pm 3.3$ \\
\hline
\end{tabular}

MDD: mean droplet diameter; TPN: total parenteral nutrition; Lipofundin ${ }^{\circledR}$ MCT/LCT-containing TPN admixtures without $(\mathrm{A})$ and with ampicillin (A + AMP). LIPIDem $^{\circledR}$-containing TPN admixtures without (B) and with ampicillin $(\mathrm{B}+\mathrm{AMP})$.

Another important parameter characterizing lipid emulsion particles is the polydispersity index (PDI), which should be in the range 0.05-0.7. Based on the results, it was found that for all tested samples the PDI expressing the degree of inhomogeneity of the system's particles was in the range from 0.05 to 0.13 , so the DLS technique was suitable for measuring the particle size of the studied TPN admixtures.

As shown in Figure 2, the zeta potential values of the reference sample $(\mathrm{A}, \mathrm{B})$ on the first day of storage differed significantly between Lipofundin ${ }^{\circledR}$ MCT/LCT-containing $(-10.55 \pm 0.76 \mathrm{mV})$ and LIPIDem ${ }^{\circledR}$-containing $(-18.63 \pm 1.03 \mathrm{mV})$ TPN admixtures. The addition of AMP resulted in a significant reduction of the zeta potential on the first day of storage $(p<0.05)$. During the study, an increase in the zeta potential was observed. When comparing the results obtained in the following hours to the value of the zeta potential measured immediately after preparation of each sample, no statistically significant differences were found for the reference samples during storage. In the case of samples containing AMP, many results showed statistically significant differences between the zeta potential at $\mathrm{t}=0 \mathrm{~h}$ (Figures 2 and 3). Interestingly, for samples stored at $25 \pm 1{ }^{\circ} \mathrm{C}$ with and without light protection, there were almost no differences between zeta potential values obtained on the following day and the value obtained on the first day.

The TPN admixtures containing vitamins and trace elements were slightly yellow, and the color deepened over time. The PDI for Lipofundin ${ }^{\circledR}$ MCT/LCT-containing and LIPIDem ${ }^{\circledR}$-containing TPN admixtures with vitamins and trace elements after preparation was 0.04 and 0.08 , respectively. The addition of AMP and 24-h storage at $25 \pm 1{ }^{\circ} \mathrm{C}$ did not affect PDI, which ranged from 0.07 to 0.09. For samples containing vitamins, trace elements, and AMP, MDDs were slightly lower compared to the corresponding samples without vitamins, trace elements, or AMP (Table 3). In the particle size distributions by volume obtained by laser diffraction method, the values of $\mathrm{Dv}(90)(90 \%$ of the particles lies below the value) were in the range of $446 \mathrm{~nm}$ to $457 \mathrm{~nm}$ for both fresh formulations and after 24-h storage. All droplet sizes were below $525 \mathrm{~nm}$ and there were no particles larger than $5 \mu \mathrm{m}$. The addition of vitamins and trace elements caused a slight decrease in $\mathrm{pH}$, whereas the values obtained on the day of preparation were maintained after $24 \mathrm{~h}$. The zeta potential values for the samples containing vitamins and trace elements without and with the addition of AMP were lower than those obtained for analogous samples without these additives (Table 3). 
Table 3. Physical characteristics of studied TPN admixtures with vitamins and trace elements. Results are shown as the mean values of three samples \pm SD.

\begin{tabular}{|c|c|c|c|c|c|c|c|c|c|}
\hline \multirow[b]{2}{*}{ Sample } & \multirow[b]{2}{*}{ Time } & \multicolumn{2}{|c|}{ DLS } & \multicolumn{4}{|c|}{ LD } & \multirow{2}{*}{$\begin{array}{l}\text { Zeta Potential } \\
\quad \pm \mathrm{SD}(\mathrm{mV})\end{array}$} & \multirow[b]{2}{*}{$\mathrm{pH} \pm \mathrm{SD}$} \\
\hline & & $\begin{array}{l}\mathrm{MDD} \pm \mathrm{SD} \\
(\mathrm{nm})\end{array}$ & $\mathrm{PDI} \pm \mathrm{SD}$ & $\operatorname{Dv}(90)(\mathrm{nm})$ & $\begin{array}{c}\text { Particles < } 500 \mathrm{~nm} \\
(\%)\end{array}$ & $\begin{array}{c}\text { Particles < } 525 \mathrm{~nm} \\
(\%)\end{array}$ & $\begin{array}{c}\text { Particles > } 5 \mu \mathrm{m} \\
(\%)\end{array}$ & & \\
\hline \multirow{2}{*}{$\mathbf{A}$} & 0 & $218.9 \pm 3.5$ & $0.06 \pm 0.02$ & 446 & 98.31 & 100 & 0 & $-10.90 \pm 0.26$ & $6.33 \pm 0.00$ \\
\hline & 24 & $213.8 \pm 2.9$ & $0.07 \pm 0.01$ & 447 & 98.20 & 100 & 0 & $-11.53 \pm 0.51$ & $6.34 \pm 0.00$ \\
\hline \multirow{2}{*}{$\mathrm{A}+\mathrm{Vit}+\mathrm{TE}$} & 0 & $209.9 \pm 1.1$ & $0.04 \pm 0.02$ & 453 & 97.70 & 100 & 0 & $-12.73 \pm 0.81$ & $6.52 \pm 0.00$ \\
\hline & 24 & $213.6 \pm 2.7$ & $0.09 \pm 0.02$ & 453 & 97.75 & 100 & 0 & $-13.57 \pm 0.85$ & $6.55 \pm 0.01$ \\
\hline \multirow[b]{2}{*}{$\mathrm{A}+\mathrm{AMP}+\mathrm{Vit}+\mathrm{TE}$} & 0 & $211.7 \pm 2.3$ & $0.08 \pm 0.02$ & 454 & 97.66 & 100 & 0 & $-15.47 \pm 0.60$ & $7.05 \pm 0.01$ \\
\hline & 24 & $216.0 \pm 3.5$ & $0.08 \pm 0.02$ & 454 & 97.66 & 100 & 0 & $-15.47 \pm 0.70$ & $6.95 \pm 0.01$ \\
\hline \multirow[b]{2}{*}{ B } & 0 & $219.3 \pm 1.7$ & $0.07 \pm 0.02$ & 455 & 98.42 & 100 & 0 & $-17.67 \pm 1.14$ & $6.43 \pm 0.01$ \\
\hline & 24 & $219.7 \pm 1.6$ & $0.08 \pm 0.02$ & 456 & 98.18 & 100 & 0 & $-19.07 \pm 0.71$ & $6.43 \pm 0.00$ \\
\hline \multirow[b]{2}{*}{$\mathrm{B}+\mathrm{Vit}+\mathrm{TE}$} & 0 & $215.4 \pm 1.6$ & $0.08 \pm 0.01$ & 457 & 98.74 & 100 & 0 & $-20.40 \pm 1.05$ & $6.47 \pm 0.01$ \\
\hline & 24 & $218.9 \pm 1.5$ & $0.07 \pm 0.03$ & 455 & 98.18 & 100 & 0 & $-20.63 \pm 0.72$ & $6.45 \pm 0.00$ \\
\hline \multirow{2}{*}{$\mathrm{B}+\mathrm{AMP}+\mathrm{Vit}+\mathrm{TE}$} & 0 & $213.9 \pm 1.4$ & $0.09 \pm 0.01$ & 457 & 98.00 & 100 & 0 & $-25.07 \pm 0.60$ & $7.00 \pm 0.00$ \\
\hline & 24 & $216.2 \pm 0.6$ & $0.08 \pm 0.00$ & 457 & 98.74 & 100 & 0 & $-23.50 \pm 1.28$ & $6.88 \pm 0.01$ \\
\hline
\end{tabular}

TPN: total parenteral nutrition; A: Lipofundin ${ }^{\circledR}$ MCT /LCT-containing TPN admixture without ampicillin; B: LIPIDem ${ }^{\circledR}$-containing TPN admixture without ampicillin; A + VIT + TE, B + VIT + TE: TPN admixtures with vitamins and trace elements; A + AMP + VIT + TE, B + AMP + VIT + TE: TPN admixtures with ampicillin, vitamins, and trace elements; MDD: mean droplet diameter; PDI: polydispersity index; DLS: dynamic light scattering; LD: laser diffraction; Dv(90): 90\% of the particles lies below the value; SD: standard deviation. 


\subsection{Chemical Stability}

AMP concentration after the first $24 \mathrm{~h}$ was found to be $92.81 \pm 0.56 \%$ and $92.97 \pm 1.10 \%$ of the zero-time concentration for Lipofundin ${ }^{\circledR}$ MCT/LCT-containing and LIPIDem ${ }^{\circledR}$-containing TPN admixtures stored at $4 \pm 1{ }^{\circ} \mathrm{C}$ (Table 4). Considering Lipofundin ${ }^{\circledR}$ MCT/LCT-containing TPN admixtures with AMP stored at room temperature (at $25 \pm 1{ }^{\circ} \mathrm{C}$ ), our results proved the impact of direct light on accelerated degradation. Samples stored without light protection were unstable even during the first $24 \mathrm{~h}(89.01 \pm 0.85 \%)$, whereas those stored at the same temperature with light protection were still within the accepted limit $(90.77 \pm 1.28 \%)$. Chromatographic data showed that prolonged storage of TPN admixtures causes a decrease in the content of AMP above the limit of $10 \%$.

Table 4. AMP content in TPN admixtures during storage.

\begin{tabular}{|c|c|c|c|c|}
\hline \multirow{4}{*}{ Time (h) } & \multicolumn{4}{|c|}{ Mean Content \pm SD (\%) } \\
\hline & \multicolumn{3}{|c|}{$\mathbf{A}+\mathbf{A M P}$} & $\mathbf{B}+\mathbf{A M P}$ \\
\hline & \multirow{2}{*}{$\begin{array}{c}4 \pm 1^{\circ} \mathrm{C} \text { with } \\
\text { Light Protection }\end{array}$} & \multicolumn{2}{|c|}{$25 \pm 1^{\circ} \mathrm{C}$} & \multirow{2}{*}{$\begin{array}{l}4 \pm 1^{\circ} \mathrm{C} \text { with } \\
\text { Light Protection }\end{array}$} \\
\hline & & $\begin{array}{l}\text { With Light } \\
\text { Protection }\end{array}$ & $\begin{array}{l}\text { Without Light } \\
\text { Protection }\end{array}$ & \\
\hline 0 & $100.00 \pm 0.47$ & $100.00 \pm 0.27$ & $100.00 \pm 0.57$ & $100.00 \pm 1.64$ \\
\hline 24 & $92.81 \pm 0.56$ & $90.77 \pm 1.28$ & $89.01 \pm 0.85$ & $92.97 \pm 1.10$ \\
\hline 48 & $88.70 \pm 1.71$ & $76.28 \pm 1.65$ & $71.55 \pm 0.46$ & $83.02 \pm 1.02$ \\
\hline 72 & $77.47 \pm 1.73$ & $71.18 \pm 1.32$ & $67.27 \pm 1.84$ & $77.20 \pm 2.82$ \\
\hline 96 & $76.19 \pm 1.63$ & $67.86 \pm 2.07$ & $64.40 \pm 0.73$ & $72.82 \pm 1.25$ \\
\hline 120 & $72.74 \pm 1.95$ & $62.02 \pm 1.30$ & $60.97 \pm 1.68$ & $67.41 \pm 1.44$ \\
\hline 144 & $66.10 \pm 1.45$ & $60.76 \pm 0.35$ & $55.30 \pm 1.86$ & $63.52 \pm 0.06$ \\
\hline
\end{tabular}

AMP: ampicillin; TPN: total parenteral nutrition; Lipofundin ${ }^{\circledR}$ MCT/LCT-containing TPN admixtures without (A) and with ampicillin (A + AMP). LIPIDem $^{\circledR}$-containing TPN admixtures without (B) and with ampicillin (B + AMP).

\section{Discussion}

There is lack of consensus on the parameters to be determined when evaluating the physical stability of TPN admixtures. Some authors suggest that the large-diameter droplet tail by single-particle optical sizing technique should be performed to proof absence of particles above $5 \mu \mathrm{m}$ [15-18]. However, we chose $\mathrm{pH}$, the particle size of the lipid emulsions by DLS and LD techniques, and the zeta potential. Such methods were used for investigating the stability of TPN admixtures by Garcia et al. [3], Mediavilla et al. [4], and Riera et al. [5]. These parameters can be used for a quick and easy assessment of the stability of lipid emulsions, such as TPN admixtures. According to literature data [15], the risk of destabilization of the oil-water system (TPN admixture) increases when the $\mathrm{pH}$ decreases below 5.5. Regarding the particle size changes of lipid emulsions administered intravenously, it should be noted that the largest endogenous lipoproteins, chylomicrons, have a diameter of 75 to $600 \mathrm{~nm}$. Thus, the particle size of lipid emulsions for parenteral administration should not exceed $500 \mathrm{~nm}$, as larger particles could cause capillary blockages and serious clinical consequences, including damage to blood vessels in the lungs, liver, and retina of the eye $[16,17]$. According to the requirements of the United States Pharmacopoeia, the average particle size of fat emulsions measured by the DLS method must be below $500 \mathrm{~nm}$ [11]. It should be emphasized that slight changes in the size of the lipid emulsion particles on consecutive storage days are a common occurrence. The TPN admixture, which is an oil-in-water system, is characterized by the constant movement of lipid emulsion particles, according to Brownian motion. The lack of clear and significant changes in particle size after the addition of AMP indicates that this drug does not significantly affect the stability of the TPN admixture regarding the stability of lipid emulsions. Another parameter characterizing lipid emulsion is zeta potential (i.e., the charge on the surface of lipid emulsion particles), allowing for the assessment of the force of electrostatic interactions between the particles. The zeta potential depends on the concentration of electrolytes and on the $\mathrm{pH}$ of the TPN admixture. It has been shown that, due to the presence of 
stabilizing phospholipids, pharmaceutical formulations of lipid emulsions used for the preparation of TPN admixtures are characterized by zeta potential values from $-40 \mathrm{mV}$ to $-50 \mathrm{mV}$, and therefore exhibit considerable stability [19]. It has also been proven that the addition of amino acid solutions to the lipid emulsion reduces the absolute value of the zeta potential by approximately $10 \mathrm{mV}$ [20-22]. Moreover, the addition of monovalent sodium, potassium, and bivalent ions of magnesium and calcium significantly reduces the zeta potential through specific and non-specific adsorption on the surface of the emulsion particles. Depending on the composition of TPN admixtures, the zeta potential may have values ranging from $-4.1 \mathrm{mV}$ to $-1.7 \mathrm{mV}$, or even close to zero [20-23]. Such a significant difference in the value of the zeta potential between the LIPIDem ${ }^{\circledR}$-containing and Lipofundin ${ }^{\circledR}$ MCT/LCT-containing TPN admixtures result from the presence of another type of lipid emulsion. LIPIDem ${ }^{\circledR}$ contains 50\% medium-chain $\mathrm{f}$ triglycerides (MCT), 40\% long-chain triglycerides (LCT), and $10 \%$ triglycerides of omega-3 acids, whereas Lipofundin ${ }^{\circledR}$ MCT /LCT $20 \%$ consists of equal amounts of MCT and LCT. On the basis of the results, it may be suggested that the addition of omega-3 acids stabilizes the oil-water system and increases the absolute value of the zeta potential. Our findings indicate that the addition of AMP causes a reduction in the zeta potential regardless of the type of lipid emulsion. However, after the reduction of zeta potential, a significant increase in its values was observed during storage, thus indicating the destabilizing effect of AMP on lipid emulsion during prolonged storage.

The HPLC method was used to assess the chemical stability of the drug in the TPN admixture. According to the criteria adopted for this study, the content of the drug in TPN should not decrease below $90 \%$ of zero-time concentration. Results above the $90 \%$ limit were observed for TPN admixtures stored at $4 \pm 1{ }^{\circ} \mathrm{C}$ and $25 \pm 1{ }^{\circ} \mathrm{C}$ with light protection but only within the first $24 \mathrm{~h}$. The standard infusion of TPN admixture lasts from 16 to $24 \mathrm{~h}$. Thus, the 24-h chemical stability of AMP in TPN admixture is sufficient for ex tempore administration.

The literature shows conflicting results for AMP compatibility tests, which might be related to the different concentrations of AMP and/or the different ratios and different types of parenteral nutrition admixtures (2-in-1 or total parenteral nutrition) applied in the studies [24]. In the compatibility studies, after Y-site administration of 2-in-1 admixtures (without lipid emulsion) precipitation was observed [1,25]. It was probably the effect of precipitating calcium phosphate when the $\mathrm{pH}$ of the TPN admixture increased above 7.2. In our study, the TPN admixtures were prepared using glycerophosphate and calcium gluconate, organic compounds that do not dissociate and do not give free calcium and phosphate ions. For this reason, the precipitation of calcium phosphate or dicalcium phosphate in a mixture of calcium gluconate and glycerophosphate is practically impossible even after exceeding the equilibrium solubility. Moreover, in another study, the administration of $20 \mathrm{mg} / \mathrm{mL}$ AMP via Y-site with nine representative TPN admixtures (containing lipid emulsion) at the ratio 1:1 was defined as compatible [26].

The results of our study can be applied both for non-supplemented and supplemented with vitamins and trace elements TPN admixtures as well as for Y-site co-administration of AMP and TPN admixtures. However, the concentration of the drug should be calculated and TPN admixture compositions taken into account. It should be noted that our study was conducted on two types of TPN admixtures differing in the source of lipid emulsion. The composition was based on the ASPEN and ESPEN guidelines. Therefore, the differences in TPN admixture compositions should be considered when evaluating the results of this study. Given that in our study we determined the stability of AMP in a dose of $10 \mathrm{~g}$ per day, which is common against meningitis, especially in neurosurgical intensive care units, the possibility of adding other doses to TPN admixtures should be confirmed by chemical stability studies.

\section{Conclusions}

The reference samples of TPN admixtures (A, B) were characterized by adequate stability during storage, regardless of the type of lipid emulsion, temperature, and light access. The observed changes 
in zeta potential and MDD of lipid emulsions were insignificant and remained within the required values. It was also observed that the addition of AMP to TPN admixture significantly reduced the zeta potential, increased the $\mathrm{pH}$ of the TPN admixtures, and slightly changed the MDD of the lipid emulsion. However, all physical stability parameters obtained in this work did not exceed the limits. For all samples (TPN admixtures, TPN admixtures with AMP, TPN admixtures with vitamins and trace elements, and TPN admixtures with vitamins, trace elements, and AMP), the MDD and Dv90 were below $500 \mathrm{~nm}$, which is in line with pharmacopoeial requirements.

Our chemical stability studies indicated that the addition of AMP to TPN admixture is possible only for ex tempore use and with light protection. The decomposition of AMP occurred in both Lipofundin ${ }^{\circledR}$ MCT/LCT-containing and LIPIDem ${ }^{\circledR}$-containing TPN admixtures, indicating that it does not depend on the composition of the fat emulsion but on the temperature and light access during storage.

Our research has demonstrated the importance of evaluating both the physical stability of TPN admixtures and the chemical stability of drugs to be added. The degradation of AMP observed during the days following its addition did not significantly affect the physical stability of the TPN admixture. It may be misleading to rely exclusively on physical stability tests due to the risk of administering a drug with a reduced active substance content and/or harmful degradation products.

Author Contributions: M.S. contributed to conception and design of the research, acquisition, analysis, interpretation of the data; A.G., K.D., E.G. and M.O. contributed to analysis, interpretation of the data; A.J. critically revised the manuscript. All authors drafted the manuscript, critically revised the manuscript, agree to be fully accountable for ensuring the integrity and accuracy of the work, and read and approved the final manuscript.

Funding: This study was supported by grant SONATA no. 2015/17/D/NZ7/00792 from the National Science Centre, Poland.

Conflicts of Interest: The authors declare no conflict of interest.

\section{References}

1. Staven, V.; Iqbal, H.; Wang, S.; Grønlie, I.; Tho, I. Physical compatibility of total parenteral nutrition and drugs in Y-site administration to children from neonates to adolescents. J. Pharm. Pharm. 2016, 69, 448-462. [CrossRef] [PubMed]

2. Staven, V.; Wang, S.; Grønlie, I.; Tho, I. Development and evaluation of a test program for Y-site compatibility testing of total parenteral nutrition and intravenous drugs. Nutr. J. 2015, 15, 29. [CrossRef] [PubMed]

3. Garcia, J.; Garg, A.; Song, Y.; Fotios, A.; Andersen, C.; Garg, S. Compatibility of intravenous ibuprofen with lipids and parenteral nutrition, for use as a continuous infusion. PLoS ONE 2018, 13, e0190577. [CrossRef] [PubMed]

4. Mediavilla, M.M.; Molina, A.; Navarro, L.; Grau, L.; Pujol, M.D.; Cardenete, J.; Cardona, D.; Riera, P. Physicochemical Compatibility of Amiodarone with Parenteral Nutrition. JPEN J. Parenter. Enter. Nutr. 2018. [CrossRef] [PubMed]

5. Riera, P.; Garrido-Alejos, G.; Cardenete, J.; Moliner, E.; Zapico-Muñiz, E.; Cardona, D.; Garin, N. Physicochemical Stability and Sterility of Standard Parenteral Nutrition Solutions and Simulated Y-Site Admixtures for Neonates. Nutr. Clin. Pract. 2018, 33, 694-700. [CrossRef] [PubMed]

6. Kanji, S.; Lam, J.; Johanson, C.; Singh, A.; Goddard, R.; Fairbairn, J.; Lloyd, T.; Monsour, D.; Kakal, J. Systematic review of physical and chemical compatibility of commonly used medications administered by continuous infusion in intensive care units. Crit. Care Med. 2010, 38, 1890-1898. [CrossRef] [PubMed]

7. Cardona, D.; Nadal, M.; Estelrich, J.; Mangues, M.A. Review of drug stability in parenteral nutrition admixtures. e-SPEN J. 2013, 8. [CrossRef]

8. Stawny, M.; Olijarczyk, R.; Jaroszkiewicz, E.; Jelińska, A. Pharmaceutical point of view on parenteral nutrition. Sci. World J. 2013, 2013. [CrossRef] [PubMed]

9. Mühlebach, S. Basics in clinical nutrition: Drugs and nutritional admixtures. e-SPEN 2009, 4. [CrossRef]

10. Mundi, M.S.; Nystrom, E.M.; Hurley, D.L.; McMahon, M.M. Management of Parenteral Nutrition in Hospitalized Adult Patients. J. Parenter. Enter. Nutr. 2017, 41, 535-549. [CrossRef] [PubMed] 
11. USP $<729>$ Globule Size Distribution in Lipid Injectable Emulsions; The United States Pharmacopeia 33/National Formulary 28; United States Pharmacopeial Convention: Rockville, MD, USA, 2009; pp. 314-316.

12. Council of Europe. European Pharmacopoeia, 9th ed.; Council of Europe: EDQM, Strasburg, France, 2017.

13. ISO 13099-1. Colloidal Systems Methods for Zeta Potential Determination Part 1: Electroacoustic and Electrokinetic Phenomena; International Organization of Standards; ISO: Geneva, Switzerland, 2012.

14. ISO 13099-2, I. Colloidal Systems Methods for Zeta Potential Determination Part 2: Optical Methods; International Organization of Standards; ISO: Geneva, Switzerland, 2012.

15. Driscoll, D.F.; Bhargava, H.N.; Li, L.; Zaim, R.H.; Babayan, V.K.; Bistrian, B.R. Physicochemical stability of total nutrient admixtures. Am. J. Heal. Pharm. 1995, 52, 623-634. [CrossRef] [PubMed]

16. Driscoll, D.F.P.; Nehne, J.P.; Peterss, H.; Klutsch, K.P.; Bistrian, B.R.M.P.; Niemann, W.P. Physical Assessments of Lipid Injectable Emulsions Via Microscopy: A Comparison to Methods Proposed in United States Pharmacopeia Chapter 729. Int. J. Pharm. Compd. 2006, 10, 309-315.

17. Driscoll, D.F. Lipid injectable emulsions: Pharmacopeial and safety issues. Pharm. Res. 2006, 23, $1959-1969$. [CrossRef] [PubMed]

18. Klang, M.G. PFAT5 and the Evolution of Lipid Admixture Stability. J. Parenter. Enter. Nutr. JPEN 2015, 39, 67S-71S. [CrossRef] [PubMed]

19. Gonyon, T.; Carter, P.W.; Dahlem, O.; Denet, A.R.; Owen, H.; Trouilly, J.L. Container effects on the physicochemical properties of parenteral lipid emulsions. Nutrition 2008, 24, 1182-1188. [CrossRef] [PubMed]

20. Washington, C. Stability of lipid emulsions for drug delivery. Adv. Drug Deliv. Rev. 1996, 20, 131-145. [CrossRef]

21. Washington, C.; Athersuch, A.; Kynoch, D.J. The electrokinetic properties of phospholipid stabilized fat emulsions. IV. The effect of glucose and of pH. Int. J. Pharm. 1990, 64, 217-222. [CrossRef]

22. Washington, C. The electrokinetic properties of phospholipid-stabilized fat emulsions. II. Droplet mobility in mixed electrolytes. Int. J. Pharm. 1990, 58, 13-17. [CrossRef]

23. Télessy, I.G.; Balogh, J.; Csempesz, F.; Szente, V.; Dredán, J.; Zelkó, R. Comparison of the physicochemical properties of MCT-containing fat emulsions in total nutrient admixtures. Colloids Surf. B Biointerfaces 2009, 72, 75-79. [CrossRef] [PubMed]

24. Robinson, C.A.; Lee, J.E. Y-Site Compatibility of Medications with Parenteral Nutrition. J. Pediatr. Pharm. 2007, 12, 53-59.

25. Watson, D. Piggyback Compatibility of Antibiotics with Pediatric Parenteral Nutrition Solutions. J. Parenter. Enter. Nutr. 1985, 9, 220-224. [CrossRef] [PubMed]

26. Trissel, L.A.; Gilbert, D.L.; Martinez, J.F.; Baker, M.B.; Walter, W.V.; Mirtallo, J.M. Compatibility of medications with 3-in-1 parenteral nutrition admixtures. J. Parenter. Enter. Nutr. 1999, 23, 67-74. [CrossRef] [PubMed] 